\title{
Research Article \\ Regions of Central Configurations in a Symmetric 4 + 1-Body Problem
}

\author{
Muhammad Shoaib \\ Department of Mathematics, University of Ha'il, P.O. Box 2440, Ha’il 81451, Saudi Arabia \\ Correspondence should be addressed to Muhammad Shoaib; safridi@gmail.com
}

Received 30 September 2014; Revised 12 January 2015; Accepted 28 January 2015

Academic Editor: Dean Hines

Copyright (C) 2015 Muhammad Shoaib. This is an open access article distributed under the Creative Commons Attribution License, which permits unrestricted use, distribution, and reproduction in any medium, provided the original work is properly cited.

\begin{abstract}
The inverse problem of central configuration of the trapezoidal 5-body problems is investigated. In this 5-body setup, one of the masses is chosen to be stationary at the center of mass of the system and four-point masses are placed on the vertices of an isosceles trapezoid with two equal masses $m_{1}=m_{4}$ at positions $\left(\mp 0.5, r_{B}\right)$ and $m_{2}=m_{3}$ at positions $\left(\mp \alpha / 2, r_{A}\right)$. The regions of central configurations where it is possible to choose positive masses are derived both analytically and numerically. It is also shown that in the complement of these regions no central configurations are possible.
\end{abstract}

\section{Introduction}

To understand the dynamics presented by a total collision of the masses or the equilibrium state of a rotating system, we are led to the concept of a central configuration. For a system to be in central configuration, the acceleration of the $i$ th mass must be proportional to its position (relative to the center of mass of the system); thus, $\ddot{r}_{i}=\lambda r_{i}$ for all $i=1,2, \ldots, n$. A central configuration can also be expressed as a critical point for the function $U^{2} I$, where $I$ is the moment of inertia. Central configuration is one of the most important and fundamental topics in the study of the few-body problem. Therefore, few-body problem in general and central configurations in particular has attracted a lot of attention over the years (see, e.g., Albouy and Llibre [1] and Shoaib and Faye [2]). The straight line solutions of the $n$-body problem were first published by Moulton [3]. Moulton arranged $n$ masses on a straight line so that they always remained collinear and then solved the problem of the values of the masses at $n$ arbitrary collinear points. Palmore $[4,5]$ presented several theorems on the classification of equilibrium points in the planar $n$-body problem.

To overcome the complexities of higher dimensions of the general $n$-body problem, various restriction methods have been used. The two most common restriction methods used are to neglect the mass of one of the bodies and introduce some kind of symmetries. Papadakis and Kanavos [6] studied the photogravitational restricted five-body problem. They study the motion of a massless particle under the gravitational attraction of four equidistant particles on a circle. More recently, Kulesza et al. [7] studied a restricted rhomboidal five-body problem. They arrange the primaries on the vertices of a rhombus and the fifth massless particle in the same plane as the primaries. Ollongren [8] studied a restricted five-body problem having three bodies of equal mass $m$ placed on the vertices of the equilateral triangle; they revolve in the plane of the triangle around their gravitational center in circular orbits under the influence of their mutual gravitational attraction; at the center a mass of $\beta m$ is present where $\beta \geq 0$. A fifth body of negligible mass compared to $m$ moves in the plane under the gravitational attraction of the other bodies. Other noteworthy studies on the restricted five-body problem include Kalvouridis [9] and Markellos et al. [10].

Another method of restriction used to study the five-body problem is the introduction of some kind of symmetries. For example, Roberts [11] discussed relative equilibria for a special case of the five-body problem. He considered a configuration which consists of four bodies at the vertices of a rhombus. The fifth body is located at the center. Mioc and Blaga [12] discussed the same problem but in the postNewtonian field of Manev. They prove the existence of monoparametric families of relative equilibria for the masses 
$\left(m_{0}, 1, m, 1, m\right)$, where $m_{0}$ is the central mass, and prove that the Manev square five-body problem admits relative equilibria regardless of the value of the mass of the central body. Albouy and Llibre [1] dealt with the central configurations of the $1+4$-body problem. They considered four equal masses on a sphere whose center is a bigger fifth mass. More recent studies on the symmetrically restricted five-body problem include Shoaib et al. [13, 14], Lee and Santoprete [15], Gidea and Llibre[16], and Marchesin and Vidal [17].

So far, in the noncollinear general four- and five-body problems the main focus has been on the common question: for a given set of masses and a fixed arrangement of bodies does a unique central configuration exist? In this paper, we ask the inverse of the question, that is, given a four- or five-body configuration, if possible, find positive masses for which it is a central configuration. Similar question has been answered by Ouyang and Xie [18] for a collinear four-body problem and by Mello and Fernandes [19] for a rhomboidal four- and five-body problems.

We consider four-point masses on the vertices of an isosceles trapezoid with two equal masses $m_{1}=m_{4}$ at positions $\left(\mp 0.5, r_{B}\right), m_{2}=m_{3}$ at positions $\left(\mp \alpha / 2, r_{A}\right)$, and $m_{0}$ at the center of mass (c.o.m). We derive, both analytically and numerically, regions of central configurations in the phase space where it is possible to choose positive masses. The rest of the paper is organized as follows. In Section 2, the equations of motion for the trapezoidal four- and five-body problems are derived. In Section 3, using both analytical and numerical techniques, the regions of central configurations for a special case of the trapezoidal five-body problem where four of the masses on the vertices of the trapezoid are taken to be equal are studied. In Section 4, the isosceles trapezoidal five-body problem in its most general form is investigated for the regions of central configurations. The regions of central configurations are given both numerically and analytically. Conclusions are given in Section 5.

\section{Equations of Motion}

The classical equation of motion for the $n$-body problem has the form

$$
m_{i} \frac{d^{2} r_{i}}{d t^{2}}=\frac{\partial U}{\partial r_{i}}=\sum_{j \neq i} \frac{m_{i} m_{j}\left(r_{j}-r_{i}\right)}{\left|r_{j}-r_{i}\right|^{3}} \quad i=1,2, \ldots, n,
$$

where the units are chosen so that the gravitational constant is equal to one, $r_{i}$ is the location vector of the $i$ th body,

$$
U=\sum_{1 \leq i<j \leq n} \frac{m_{i} m_{j}}{\left|r_{j}-r_{i}\right|}
$$

is the self-potential, and $m_{i}$ is the mass of the $i$ th body.

A central configuration is a particular configuration of the $n$-bodies where the acceleration vector of each body is proportional to its position vector, and the constant of proportionality is the same for the $n$-bodies; therefore,

$$
\sum_{j=1, j \neq i}^{n} \frac{m_{j}\left(r_{j}-r_{k}\right)}{\left|r_{j}-r_{k}\right|^{3}}=-\lambda\left(r_{k}-c\right) \quad k=1,2, \ldots, n,
$$

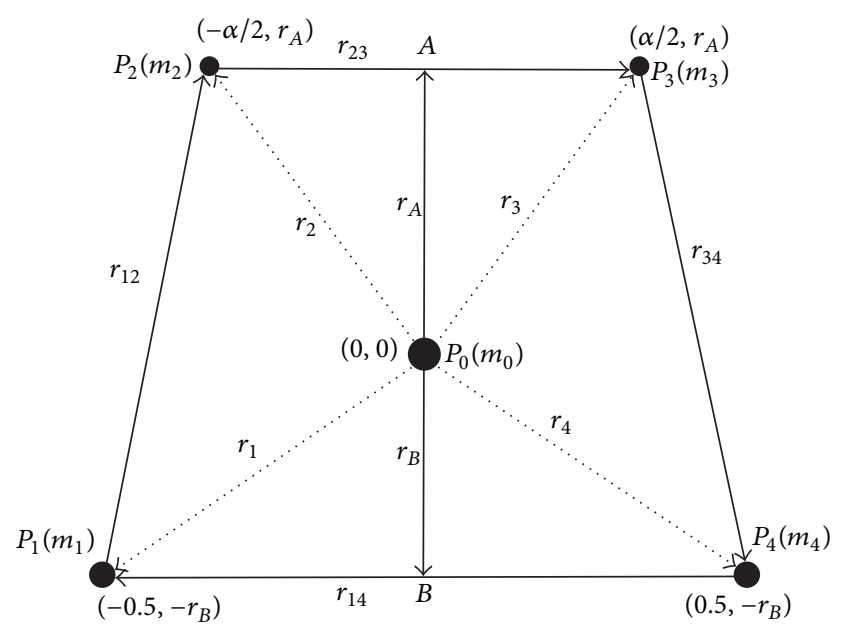

FIgURE 1: Trapezoidal four- and five-body configurations.

where

$$
\begin{gathered}
c=\frac{C}{M_{t}}, \quad C=m_{1} r_{1}+m_{2} r_{2}+\cdots+m_{n} r_{n}, \\
\lambda=\frac{U}{2 I}, \quad I=\frac{1}{2} \sum_{i=1}^{n} m_{i}\left\|r_{i}\right\|^{2} .
\end{gathered}
$$

Let us consider five bodies of masses $m_{0}, m_{1}, m_{2}, m_{3}$, and $m_{4}$. The mass $m_{0}$ is stationary at the c.o.m of the system. The remaining four bodies are placed at the vertices of an isosceles trapezoid shown in Figure 1. The geometry of the system is taken to be symmetric about the $y$-axis. As shown in Figure 1, $A$ is the center of mass of $m_{2}$ and $m_{3}$, and $B$ is the c.o.m of the masses $m_{1}$ and $m_{4}$. Because of the symmetry about $y$-axis, the symmetric masses will be equal. Therefore, we take $m_{2}=$ $m_{3}=m$ and $m_{1}=m_{4}=M$. We choose the coordinates for the five bodies as follows:

$$
\begin{gathered}
r_{0}=(0,0), \quad r_{1}=\left(-0.5,-r_{B}\right), \quad r_{2}=\left(-\frac{\alpha}{2}, r_{A}\right), \\
r_{3}=\left(\frac{\alpha}{2}, r_{A}\right), \quad r_{4}=\left(0.5,-r_{B}\right),
\end{gathered}
$$

where $r_{A}$ is the distance from the c.o.m of the system to the center of mass of $m_{2}$ and $m_{3}$ and $r_{B}$ is the distance from the c.o.m of the system to the c.o.m of $m_{1}$ and $m_{4}$.

Without loss of generality, it is assumed that $r_{23}=-\alpha r_{41}$, $r_{B A}=\left|r_{A}-r_{B}\right|=\beta r_{41}$. Using these assumptions with (1) we obtain the following equations of motion:

$$
\begin{aligned}
& \ddot{r}_{1}=-\frac{m_{0} r_{1}}{d}+\frac{m r_{12}}{a}+M r_{14}+\frac{m r_{13}}{b}, \\
& \ddot{r}_{2}=-\frac{m_{0} r_{2}}{c}+\frac{M r_{21}}{a}+\frac{m r_{23}}{\alpha^{3}}+\frac{M r_{24}}{b}, \\
& \ddot{r}_{3}=-\frac{m_{0} r_{3}}{c}+\frac{M r_{31}}{b}+\frac{m r_{32}}{\alpha^{3}}+\frac{M r_{34}}{a}, \\
& \ddot{r}_{4}=-\frac{m_{0} r_{4}}{d}+\frac{m r_{42}}{b}+M r_{41}+\frac{m r_{43}}{a},
\end{aligned}
$$


where

$$
\begin{array}{ll}
a=\left(\left(\frac{1}{2}-\frac{\alpha}{2}\right)^{2}+\beta^{2}\right)^{3 / 2}, & b=\left(\left(\frac{1}{2}+\frac{\alpha}{2}\right)^{2}+\beta^{2}\right)^{3 / 2}, \\
c=\left(\frac{\alpha^{2}}{4}+\frac{M^{2} \beta^{2}}{(m+M)^{2}}\right)^{3 / 2}, & d=\left(\frac{1}{4}+\frac{m^{2} \beta^{2}}{(m+M)^{2}}\right)^{3 / 2} .
\end{array}
$$

As $m_{0}$ is taken to be stationary at the c.o.m, therefore $\ddot{r}_{0}=0$. The c.o.m is at the origin; therefore, $r_{0}=0$. Hence, the CC equation $\ddot{r}_{0}=-\lambda r_{0}$ will simply be $0=0$ which is why we do not have a fifth equation. Let $r=r_{A}-r_{B}$ (Figure 1) and then using the geometry of our proposed problem we arrive at the following relationships between $r_{i}, i=1,2,3,4, r$, and $r_{41}$ :

$$
\begin{aligned}
& r_{1}=-\frac{m}{M+m} r+\frac{1}{2} r_{41}, \\
& r_{2}=\frac{M}{m+M} r+\frac{\alpha}{2} r_{41}, \\
& r_{3}=\frac{M}{m+M} r-\frac{\alpha}{2} r_{41}, \\
& r_{4}=-\frac{m}{M+m} r-\frac{1}{2} r_{41} .
\end{aligned}
$$

This clearly shows that it is enough to study the equations for $r=((m+M) / 2 M)\left(r_{2}+r_{3}\right)$ and $r_{41}$ as $r_{i}, i=1,2,3,4$, are linear combination of $r$ and $r_{41}$ :

$$
\begin{gathered}
\ddot{r}_{41}=\left[-2 M-\frac{m_{0}}{d}+\frac{m}{a}(\alpha-1)-\frac{m}{b}(\alpha+1)\right] r_{41}, \\
\ddot{r}_{3 p 2}=-\left[\frac{m_{0}}{c}+\frac{m+M}{a}+\frac{m+M}{b}\right]\left(r_{2}+r_{3}\right) .
\end{gathered}
$$

Using (3) in conjunction with (9) and taking the center of mass at the origin, we obtain the following equations of central configurations for the trapezoidal five-body problem:

$$
\begin{gathered}
2 M-\frac{m(\alpha-1)}{a}+\frac{m(\alpha+1)}{b}+\frac{m_{0}}{d}=\lambda, \\
\frac{m_{0}}{c}+\frac{m+M}{a}+\frac{m+M}{b}=\lambda .
\end{gathered}
$$

To obtain regions of central configurations in the trapezoidal five-body problems we will need to simultaneously solve (10) and (11) for $m$ and $M$. This will give values of the two masses which define the central configurations for the trapezoidal five-body problem.

\section{Four Equal Masses with a Varying Central Mass}

Let the four bodies on the vertices of the trapezoid have equal masses; that is, $m_{i}=M, i=1,2,3,4$ and $\lambda=1$. The equations of central configuration, given in (10) and (11), become

$$
\begin{gathered}
\left(2-\frac{(\alpha-1)}{a}+\frac{(\alpha+1)}{b}\right) M+\frac{m_{0}}{e}=\lambda, \\
2\left(\frac{1}{a}+\frac{1}{b}\right) M+\frac{m_{0}}{f}=\lambda,
\end{gathered}
$$

where

$$
e=\left(\frac{1}{4}+\frac{\beta^{2}}{4}\right)^{3 / 2}, \quad f=\left(\frac{\alpha^{2}}{4}+\frac{\beta^{2}}{4}\right)^{3 / 2}
$$

The above two equations give $m_{0}$ and $M$ as functions of $\alpha$ and $\beta$ :

$$
M=\frac{a b(f-e)}{g(\alpha, \beta)}, \quad m_{0}=\frac{e f h(\alpha, \beta)}{g(\alpha, \beta)},
$$

where

$$
\begin{aligned}
& g(\alpha, \beta)=b(2 f+e(\alpha-1))+a(2 f-e(1+2 b+\alpha)), \\
& h(\alpha, \beta)=a+b-2 a b-\alpha(a-b) .
\end{aligned}
$$

To find regions of central configurations in the $\alpha \beta$-plane where $\left(M, m_{0}\right)>(0,0)$, the sign analysis of $(f-e), g(\alpha, \beta)$, and $h(\alpha, \beta)$ is needed which is given below.

(i) As $\alpha \in(0,1), \alpha^{2}<1$; therefore, $e>f$ for all values of $\alpha$ and $\beta$ which means $f-e<0$.

(ii) For sign analysis of $h(\alpha, \beta)$, we obtain the following region where $h(\alpha, \beta)<0$ :

$$
R_{1}=\left\{(\alpha, \beta) \mid 0<\alpha<1, \beta>0, b<\frac{a(\alpha-1)}{1+\alpha-2 a}\right\}
$$

Ideally we would like to find a region which is explicitly defined by a function of $\alpha$ or $\beta$. Because of the involvement of radicals it is impossible to find a closed form solution of $h(\alpha, \beta)<0$ in terms of $\alpha$ or $\beta$. Therefore, we approximate it by a polynomial of order 2 in $\alpha$ :

$$
\begin{aligned}
h_{\mathrm{app}} \approx & -2 \beta^{6}-1.5 \beta^{4}+2(J-0.19) \beta^{2}+0.5 J \\
& +0.03\left(1.5 \beta^{4}-\left(2.25 \beta^{2}+0.75\right) J^{-1}-0.09\right) \alpha^{2} \\
& +O\left(\alpha^{3}\right)
\end{aligned}
$$

where $J=\sqrt{\beta^{2}+0.25}$. The equation $h(\alpha, \beta)_{\text {app }}=0$ gives $\alpha$ as a function $\beta$ which provides a boundary between $h<0$ and $h>0$ :

$\alpha_{1}(\beta)$

$$
\begin{aligned}
& \approx 1.15 \sqrt{\frac{J\left(-\beta^{6}-0.75 \beta^{4}+(J-0.18) \beta^{2}+0.25 J-0.02\right)}{J \beta^{4}-1.5 \beta^{2}-0.07 J-0.5}} \\
& =K_{1}(\beta) .
\end{aligned}
$$

Therefore, region $R_{1}$ can now be rewritten as below:

$$
R_{1} \approx\left\{(\alpha, \beta) \mid \beta>0, \alpha(\beta)<K_{1}(\beta)\right\} .
$$

It is numerically verified that $h(\alpha, \beta)=0$ and $h(\alpha$, $\beta)_{\text {app }}=0$ have almost identical graphs for all values of $\alpha$ and $\beta$ (see Figure 2). Region $R_{1}$ is given in Figure 2 . 


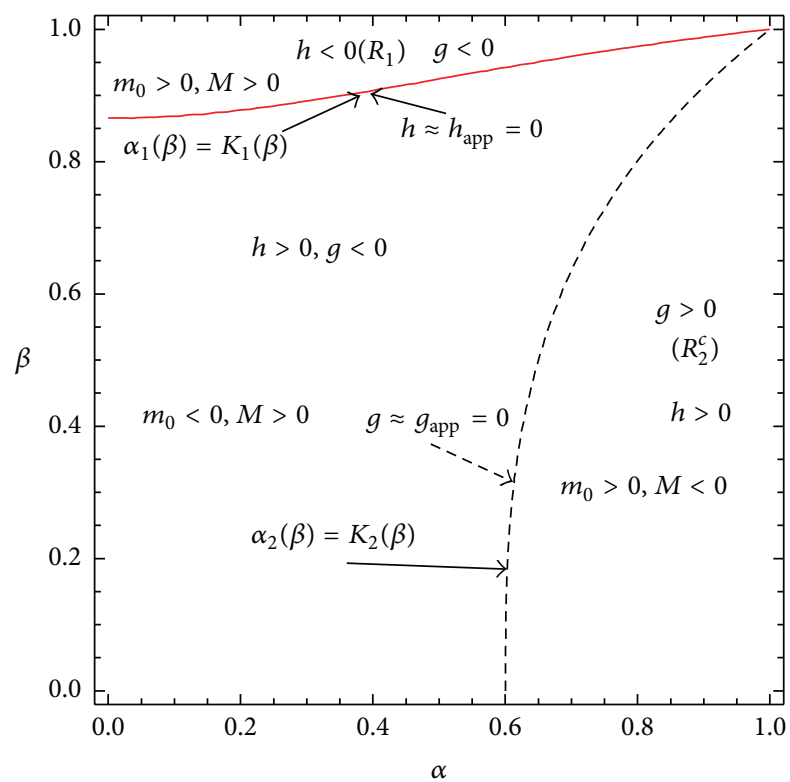

FIGURE 2: CC regions in trapezoidal 5-BP with four equal masses and a varying central mass.

(iii) For sign analysis of $g(\alpha, \beta)$, we obtain the following region where $g(\alpha, \beta)<0$ :

$R_{2}=\left\{(\alpha, \beta) \mid 0<\alpha<1, \beta>0, b<\frac{a(2 f+e(\alpha+1))}{2 f-2 a e+e(\alpha-1)}\right\}$

To find the region $g(\alpha, \beta)<0$ which is explicitly defined by a function of $\alpha$ or $\beta$ we approximate $g(\alpha, \beta)$ by a polynomial of order 2 in $\alpha$ :

$$
\begin{aligned}
& g(\alpha, \beta)_{\mathrm{app}} \\
& =-0.77 \beta^{6}+0.38 \beta^{5}-0.74 \beta^{4}+0.06 \beta^{3}-0.3 \beta^{2} \\
& -0.04+J^{-1}\left(0.08 \beta+1.5 \beta^{5}+0.75 \beta^{3}\right. \\
& \left.\quad-0.3 J \sqrt{\beta^{2}+1}\left(\beta^{6}+\beta^{4}-0.36\right)\right) \alpha^{2} .
\end{aligned}
$$

The real valued function $g_{\text {app }}(\alpha, \beta)=0$ gives $\alpha$ as a function $\beta$ which provides a boundary between $g<0$ and $g>0$ :

$$
\begin{gathered}
\alpha_{2} \approx\left(\left(J \left(0.77 \beta^{6}-0.38 \beta^{5}+0.74 \beta^{4}-0.06 \beta^{3}+0.3 \beta^{2}\right.\right.\right. \\
+0.04)) \cdot\left(0.08 \beta+1.5 \beta^{5}+0.75 \beta^{3}\right. \\
\left.\left.\quad-0.3 J \sqrt{\beta^{2}+1}\left(\beta^{6}+\beta^{4}-0.36\right)\right)^{-1}\right)^{1 / 2}
\end{gathered}
$$$$
=K_{2}(\beta) \text {. }
$$

In the same way as in the case of $h(\alpha, \beta)$, it is numerically verified that $g(\alpha, \beta)=0$ and $g(\alpha, \beta)_{\text {app }}=0$ have almost identical graphs for all values of $\alpha$ and $\beta$ (see Figure 2). Therefore, region $R_{2}$ can now be rewritten as below:

$$
R_{2} \approx\left\{(\alpha, \beta) \mid \beta>0, \alpha_{2}(\beta)<K_{2}(\beta)\right\} .
$$

Numerically, region $R_{2}$ is given in Figure 2 .

As the numerator of $M$ is negative for all values of $\alpha$ and $\beta$, therefore $R_{2}$ defines a central configuration region in the $\alpha \beta$ plane where $M>0$. Similarly $m_{0}>0$ in $\left(R_{1} \cap R_{2}\right) \cup\left(R_{1}^{c} \cap R_{2}^{c}\right)$. As $R_{2}^{c}=\phi$ and $R_{1} \subset R_{2}$, therefore

$$
R_{3}=\left(R_{1} \cap R_{2}\right)=R_{1}
$$

defines the CC region for this particular setup of the 5body problem where all the masses are positive. Numerically, regions $R_{1}, R_{2}$, and $R_{3}$ are shown in Figure 2. The Central Configuration regions where $m_{0}>0$ and $M>0$ are given in Figure 3.

\section{Two Pairs of Equal Masses and a Central Mass}

As stated earlier the geometry in Figure 1 is taken to be symmetric about the line $A B$, where $A$ is the center of mass of $m_{2}$ and $m_{3}$, and $B$ is the center of mass of $m_{1}$ and $m_{4}$. Let $m_{1}=m_{4}=M$ and $m_{2}=m_{3}=m$. We further assume that $m \leq M, \lambda=1$, and $m=C M$, where $0<C \leq 1$. Therefore, (9) give us

$$
\begin{gathered}
\frac{M(a(-2 b-C(\alpha+1))+b C(\alpha-1))}{a b}-\frac{m_{0}}{e}=-1, \\
\frac{2 \beta\left(a\left(b m_{0}+(C+1) f M\right)+b(C+1) f M\right)}{a b(1-C) f}=\frac{2 \beta}{1-C},
\end{gathered}
$$

where

$$
e^{*}=\left(\frac{1}{4}+\frac{C^{2} \beta^{2}}{(1+C)^{2}}\right)^{3 / 2}, \quad f^{*}=\left(\frac{\alpha^{2}}{4}+\frac{\beta^{2}}{(1+C)^{2}}\right)^{3 / 2} \text {. }
$$

To obtain regions of central configurations in the trapezoidal five-body problem we simultaneously solve the above equation for $m_{0}$ and $M$ :

$$
\begin{gathered}
M(\alpha, \beta, C)=\frac{a b\left(e^{*}-f^{*}\right)}{D_{m}}, \\
m_{0}(\alpha, \beta, C)=\frac{e^{*} f^{*} N_{m_{0}}}{D_{m}},
\end{gathered}
$$

where

$$
\begin{gathered}
N_{m_{0}}(\alpha, \beta, C)=\alpha C(a-b)+2 a b-a-b \\
D_{m}(\alpha, \beta, C)=\alpha C e^{*}(a-b)+(a+b)\left(C e^{*}-f^{*}(C+1)\right) \\
+2 a b e^{*} .
\end{gathered}
$$




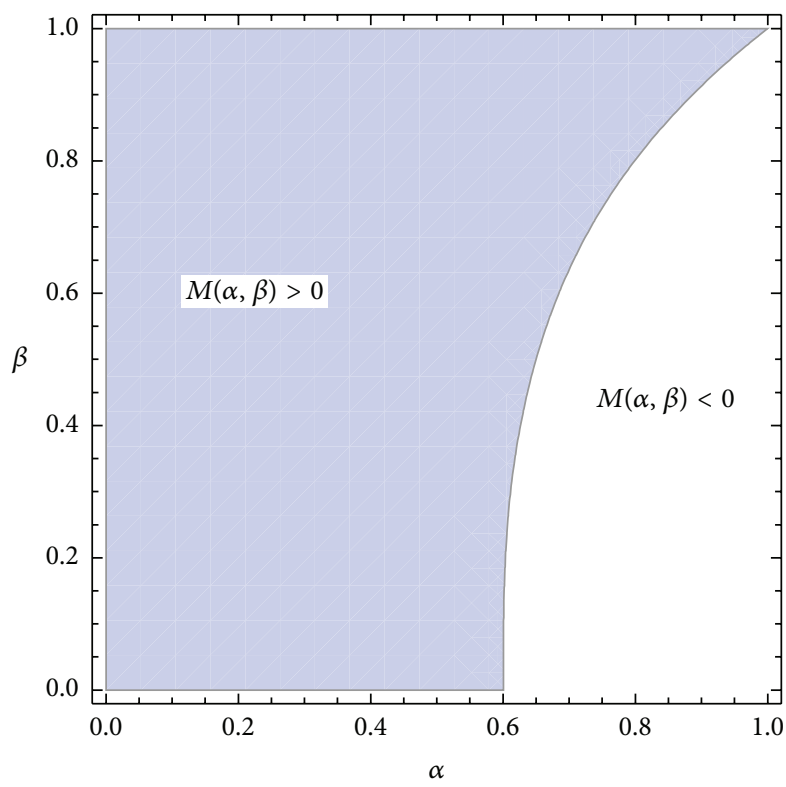

(a)

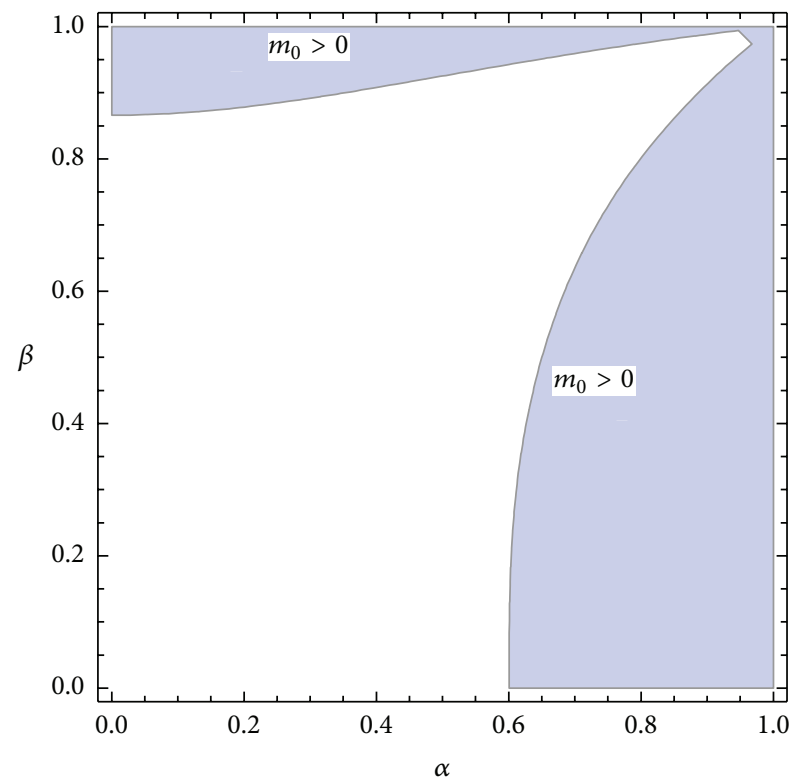

(b)

Figure 3: (a) $M>0$ (colored) (b) $m_{0}>0$ (colored).

The above equations representing $M$ and $m_{0}$ give regions of central configurations for the trapezoidal five-body problem in the $\alpha \beta C$-plane. For example, when (1) $\alpha=0.2, \beta=0.8$, and $C=0.3,\left(M, m_{0}\right)=(0.26,0.04)$, and when $(2) \alpha=0.4$, $\beta=0.7$, and $C=0.5,\left(M, m_{0}\right)=(-0.5,0.4)$. As can be seen in number (2), one of the masses has become negative for specific values of $\alpha$ and $\beta$, which is impractical. Therefore, we would like to identify compact regions in the central configuration space where none of the masses can become negative. The sign analysis of $\left(e^{*}-f^{*}\right), D_{m}(\alpha, \beta, C)$, and $N_{m}(\alpha, \beta, C)$ is needed which is given below.

(1) $e^{*}-f^{*}>0$ : it is straightforward to show that $e^{*}-$ $f^{*}>0$ in

$$
R_{4}=R_{4 a} \cup R_{4 b} \cup R_{4 c},
$$

where

$$
\begin{gathered}
R_{4 a}=\{(\alpha, \beta, C) \mid 0<C \leq 0.6,0<\alpha<1, \\
\left.0.5 \sqrt{\frac{\alpha^{2}(1+C)-C-1}{C-1}}<\beta<1\right\}, \\
R_{4 b}=\left\{(\alpha, \beta, C) \mid 0.6<C<1,0<\alpha<\sqrt{\frac{5 C-3}{C+1},}\right. \\
R_{4 c}=\left\{(\alpha, \beta, C) \mid 0.6<C<1, \sqrt{\frac{5 C-3}{C+1}}<\alpha<1,\right. \\
\left.0<\beta<0.5 \sqrt{\frac{\alpha^{2}(1+C)-C-1}{C-1}}\right\} .
\end{gathered}
$$

\section{Region $R_{4}$ is given in Figure 4(a):}

(2) The analysis of $N_{m_{0}}(\alpha, \beta, C)$ is similar in nature to $h(\alpha, \beta)$ but it is comparatively easier to write a closed form solution of $N_{m_{0}}(\alpha, \beta, C)=0$ as $C(\alpha, \beta)=(a+$ $b-2 a b) /(\alpha(a-b))$. Therefore, $N_{m_{0}}(\alpha, \beta, C)>0$ in the following region:

$R_{5}=\left\{(\alpha, \beta, C) \mid \frac{(a+b-2 a b)}{\alpha(a-b)}<C<1, \beta>0,0<\alpha<1\right\}$.

Like in the four equal masses case we can write a polynomial approximation of $N_{m}(\alpha, \beta, C)$ as follows:

$$
\begin{aligned}
& N_{m_{\text {oapp }}}(\alpha, \beta, C) \\
& \quad \approx\left(1.5 \beta^{4}-\sqrt{J}\left(1.5 C-0.75 \beta^{2} J^{-1}-0.375 J^{-1}\right)-0.1\right) \alpha^{2} \\
& \quad+\left(2 \beta^{6}+1.5 \beta^{4}+0.375 \beta^{2}-\sqrt{J}\left(2 \beta^{2}+0.5\right)+0.03\right) .
\end{aligned}
$$

Equation $N_{m_{\text {oapp }}}(\alpha, \beta, C)=0$ will give $\alpha$ as a function of $\beta$ and $C$ which will provide a boundary between the regions where $N_{m_{\text {oapp }}}<0$ and $N_{m_{\text {oapp }}}>0$ :

$\alpha_{3}(\beta, C)$

$$
\begin{aligned}
& \approx \sqrt{\frac{-2 \beta^{6}-1.5 \beta^{4}-0.375 \beta^{2}+\sqrt{J}\left(2 \beta^{2}+0.5\right)-0.03}{1.5 \beta^{4}-\sqrt{J}\left(1.5 C-0.75 \beta^{2} J^{-1}-0.375 J^{-1}\right)}} \\
& =K_{3}(\beta, C) .
\end{aligned}
$$




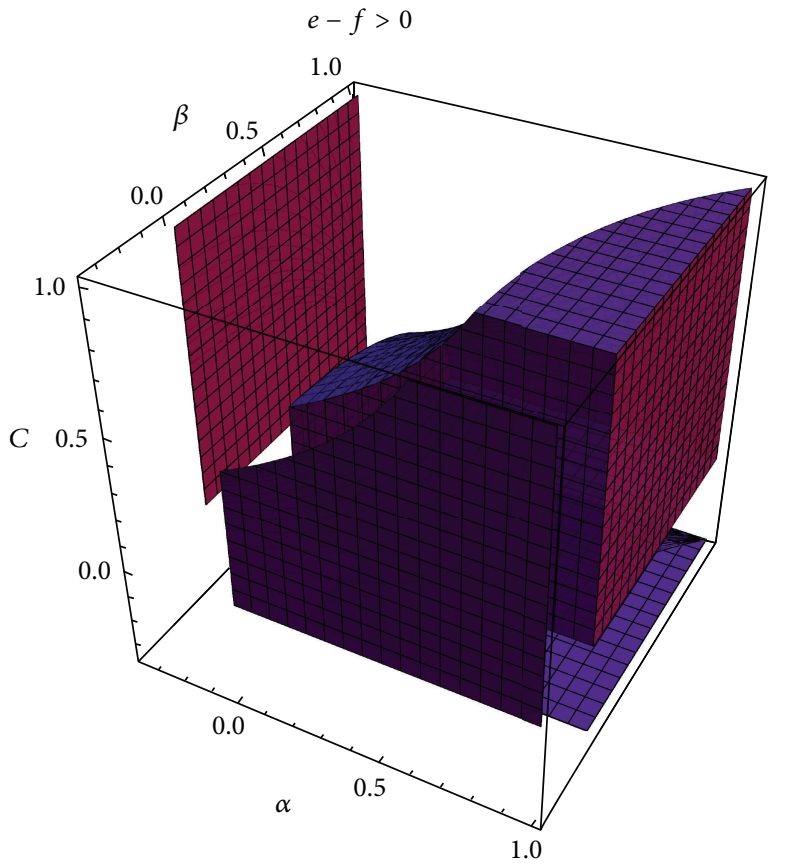

(a)

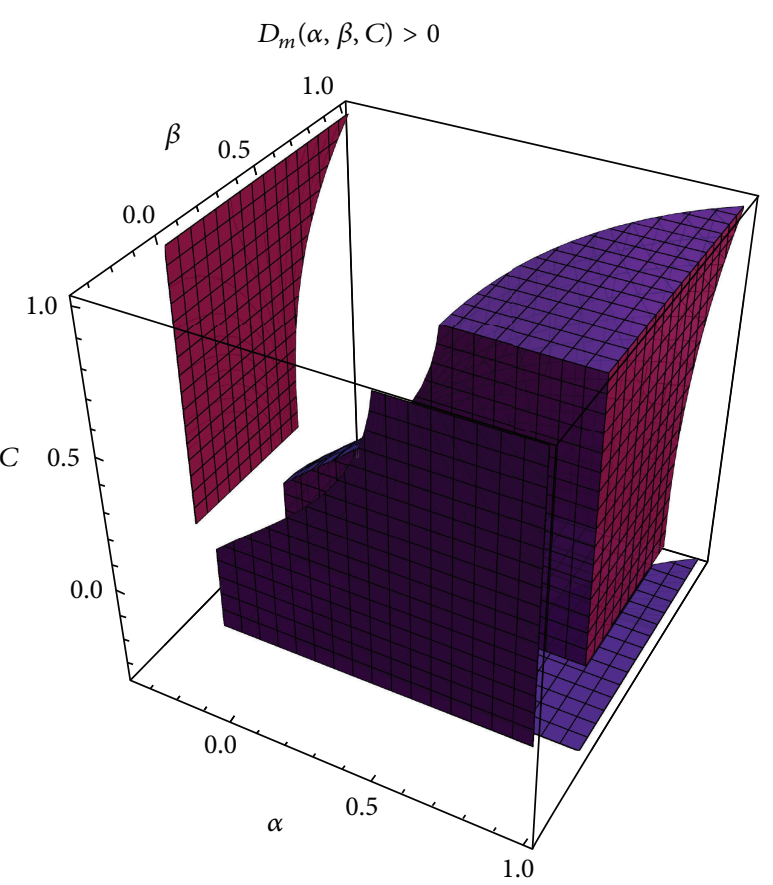

(b)

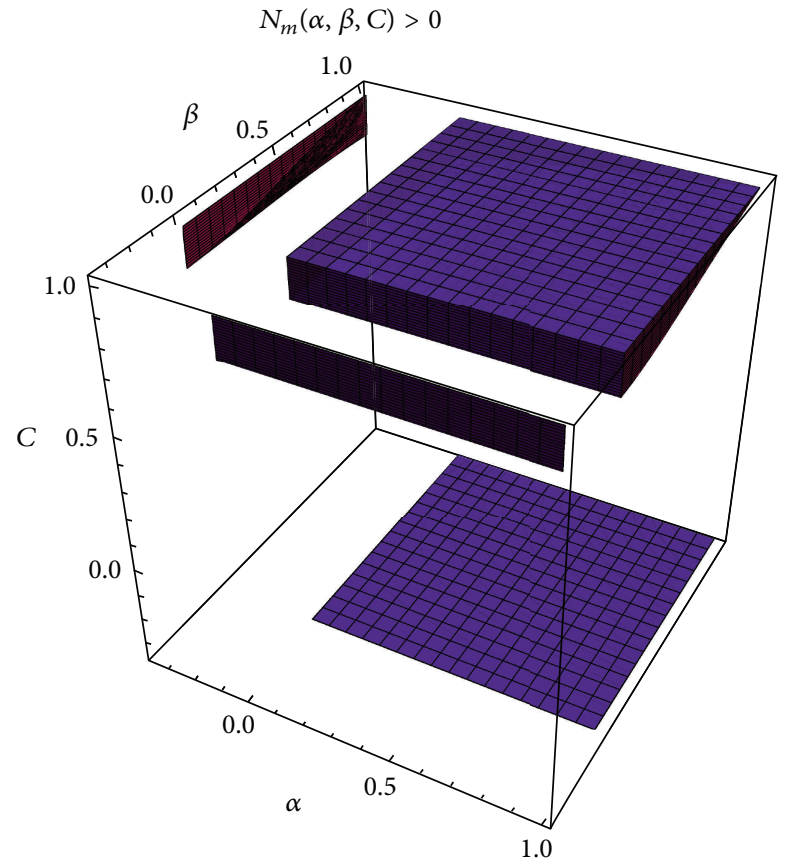

(c)

Figure 4: (a) $(e-f)(\alpha, \beta, C)>0$ (colored). (b) $D_{m}(\alpha, \beta, C)>0$ (colored). (c) $N_{m}(\alpha, \beta, C)>0$ (colored).

Region $R_{5}$ can now be rewritten as

$R_{5}=\left\{(\alpha, \beta, C) \mid 0<C<1, \beta>0,0<\alpha<K_{3}(\beta, C)\right\}$.

It is worth mentioning here that the approximation error between $N_{m_{0}}$ and $N_{m_{0 a p p}}$ is almost zero. Region $R_{5}$ is given in Figure 4(c).
(3) It is similarly easy to write $C$ as a function $\alpha$ and $\beta$ from $D_{m}(\alpha, \beta, C)=0$. Therefore, $D_{m}$ is positive in the following region:

$$
R_{6}=\left\{(\alpha, \beta, C) \mid \frac{f^{*}(a+b)-2 a b e^{*}}{(a-b)\left(e^{*}-f^{*}\right)+e^{*} \alpha(a-b)}<C<1,\right.
$$

$$
\beta>0,0<\alpha<1\} \text {. }
$$




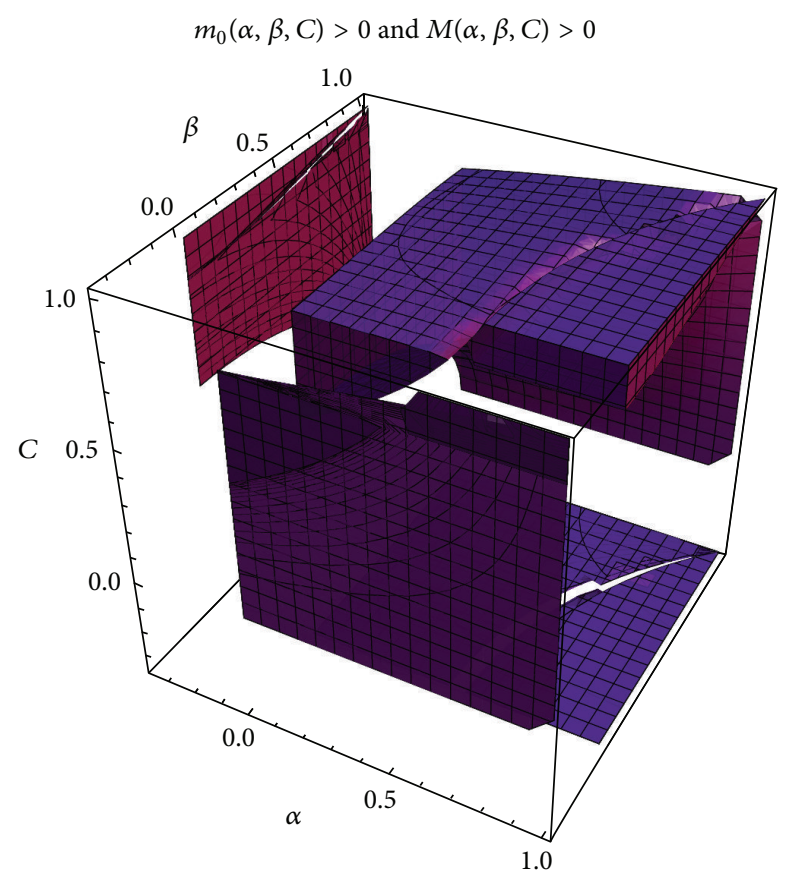

Figure 5: Central configurations region in the isosceles trapezoid 4 + 1-body problem.

The polynomial approximation of $D_{m}(\alpha, \beta, C)$ is given as below:

$$
\begin{aligned}
& D_{m_{\text {app }}}(\alpha, \beta, C) \\
& \approx J^{4}\left(1.5 C e^{*} 2 e^{*} J^{2}-\beta^{2}\left(-C^{3} \sqrt[3]{e^{*}}+\beta\right)(C+1)^{-2}\right) \\
& \quad+0.68 C_{2} \alpha^{2},
\end{aligned}
$$

where

$$
\begin{aligned}
C_{2}= & (-1.5 C e J-\beta) J^{2}+e^{*^{2 / 3}}\left(0.62+0.75 C\left(1+C J^{2}\right)\right) \\
& \cdot\left(-0.06+\beta^{4}\right)\left(1+6 J^{2} C e^{*^{1 / 3}}\right)(C+1)^{-2} .
\end{aligned}
$$

As above $D_{m_{\text {app }}}(\alpha, \beta, C)=0$ will give $\alpha$ as a function of $\beta$ and $C$ which will provide a boundary between the regions where $D_{m_{\text {app }}}<0$ and $D_{m_{\text {app }}}>0$ :

$$
\begin{gathered}
\alpha_{3}(\beta, C) \approx J^{2}\left(\frac { 1 } { C _ { 2 } } \left(-1.5 C e^{*} 2 e^{*} J^{2}+\beta^{2}\left(-C^{3} \sqrt[3]{e^{*}}+\beta\right)\right.\right. \\
\left.\left.\cdot(C+1)^{-2}\right)-0.68 C_{2} \alpha^{2}\right)^{1 / 2} \\
=K_{4}(\beta, C) .
\end{gathered}
$$

Region $R_{6}$ can now be rewritten as

$$
R_{6}=\left\{(\alpha, \beta, C) \mid 0<C<1, \beta>0,0<\alpha<K_{4}(\beta, C)\right\} .
$$

Region $R_{6}$ is given in Figure 4(b).

From the above analysis we conclude that the $\mathrm{CC}$ region where $M>0$ is

$$
R_{7}=\left(R_{4} \cap R_{6}\right) \cup\left(R_{4}^{c} \cap R_{6}^{c}\right) .
$$

Similarly, the CC region where $m_{0}>0$ is

$$
R_{8}=\left(R_{5} \cap R_{6}\right) \cup\left(R_{5}^{c} \cap R_{6}^{c}\right) .
$$

This gives the CC region for this particular setup of the fivebody problem as

$$
R_{9}=R_{7} \cap R_{8} .
$$

Region $R_{9}$ is given in Figure 5. In the complement of this region no central configurations are possible.

Hence, the central configuration region for the isosceles trapezoidal five-body problem with two pairs of masses and a stationary central mass is determined by $R_{7}$ and $R_{8}$ and is given by $R_{9}=R_{7} \cap R_{8}$. Numerically, region $R_{9}$ is shown by the colored part of Figure 5. To aid the understanding of the central configuration region $R_{9}$ its cross sections are given in Figures 6 and 7 for various values of $C$.

To complete the analysis of the $4+1$-body case with two pairs of masses we study a special case where $\alpha=\beta$. This reduces the number of parameters from three to two while we 


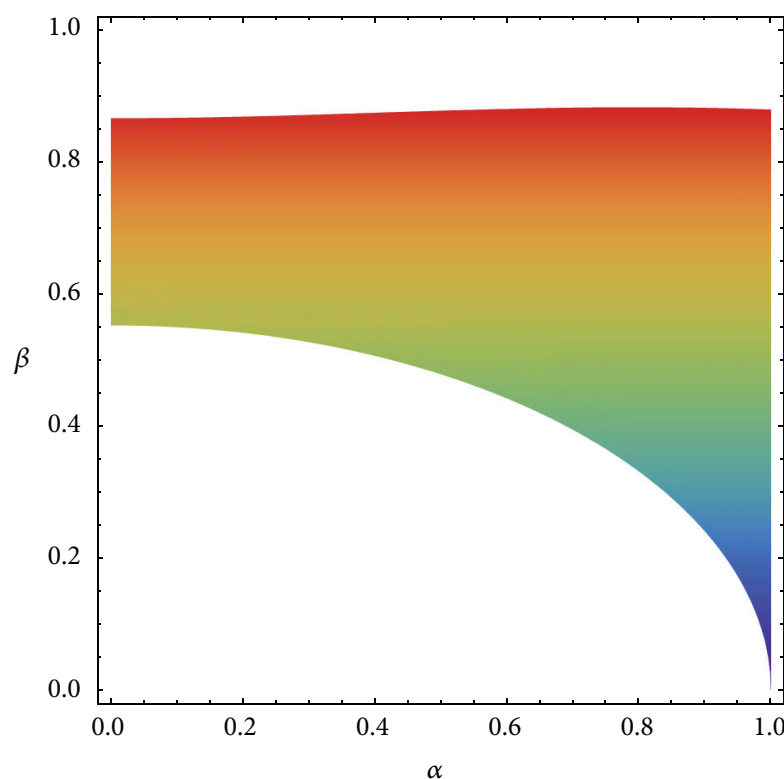

(a)

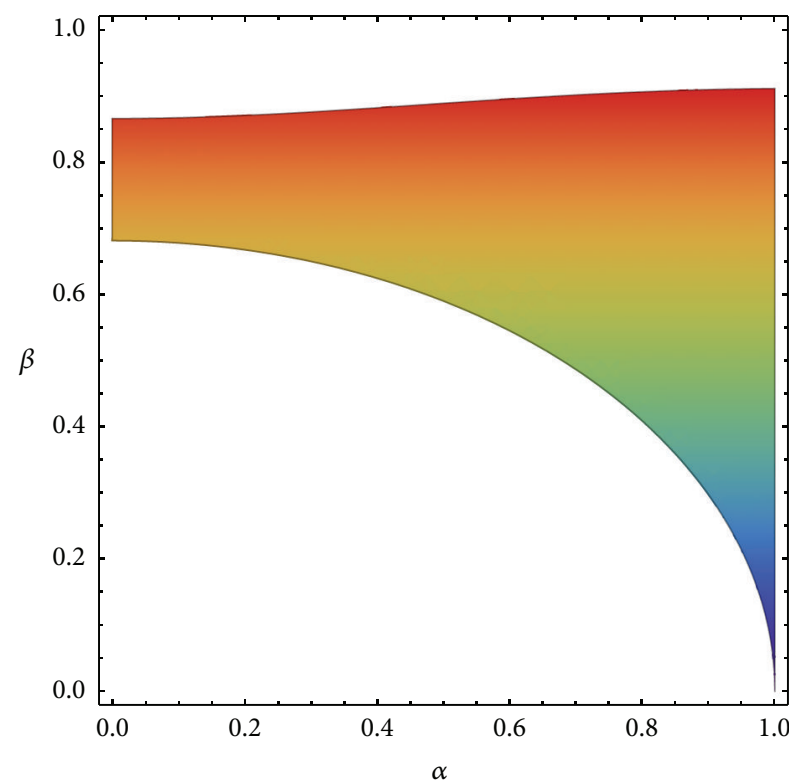

(b)

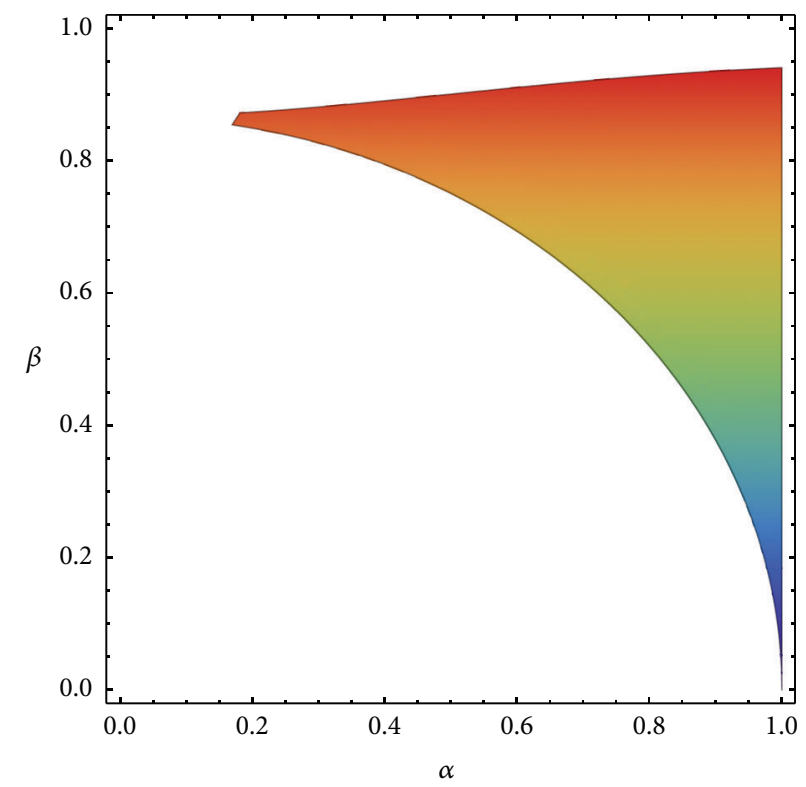

(c)

FIGURE 6: Cross sections of the central configurations region in the general case when $M, m_{0}>0$ and (a) $C=0.1$, (b) $C=0.3$, and (c) $C=0.5$.

still maintain the general setup with two pairs of masses. The $\mathrm{CC}$ regions are given only numerically in Figure 8 as it is easy to derive the analytical expressions from (25) by replacing $a, b, e, f$ with the following new values:

$$
\begin{aligned}
& a^{*}=1.4\left(0.2-0.4 \beta+\beta^{2}\right)^{3 / 2}, \\
& b^{*}=1.4\left(0.2+0.4 \beta+\beta^{2}\right)^{3 / 2},
\end{aligned}
$$

$$
\begin{aligned}
& e_{*}=\left(\frac{\beta^{2} C^{2}}{(C+1)^{2}}+0.25\right)^{3 / 2}, \\
& f_{*}=\frac{\beta^{3}}{8}\left(\frac{4}{(C+1)^{2}}+1\right)^{3 / 2} .
\end{aligned}
$$

Using $a^{*}, b^{*}, e_{*}$, and $f_{*}$, CC regions are given for $\alpha=\beta$ in Figure 8. It is obvious from Figure 8(c) that no central 


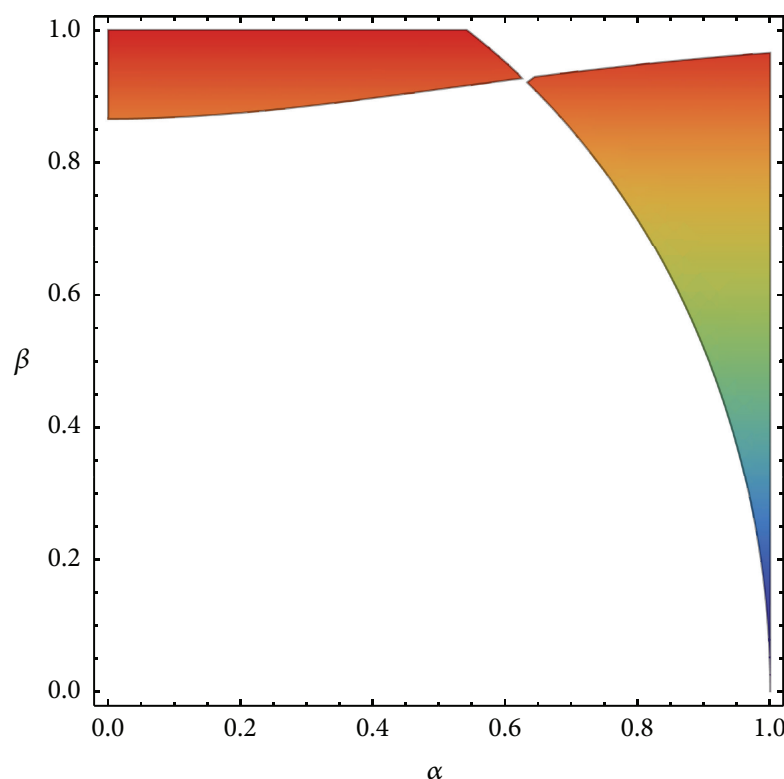

(a)

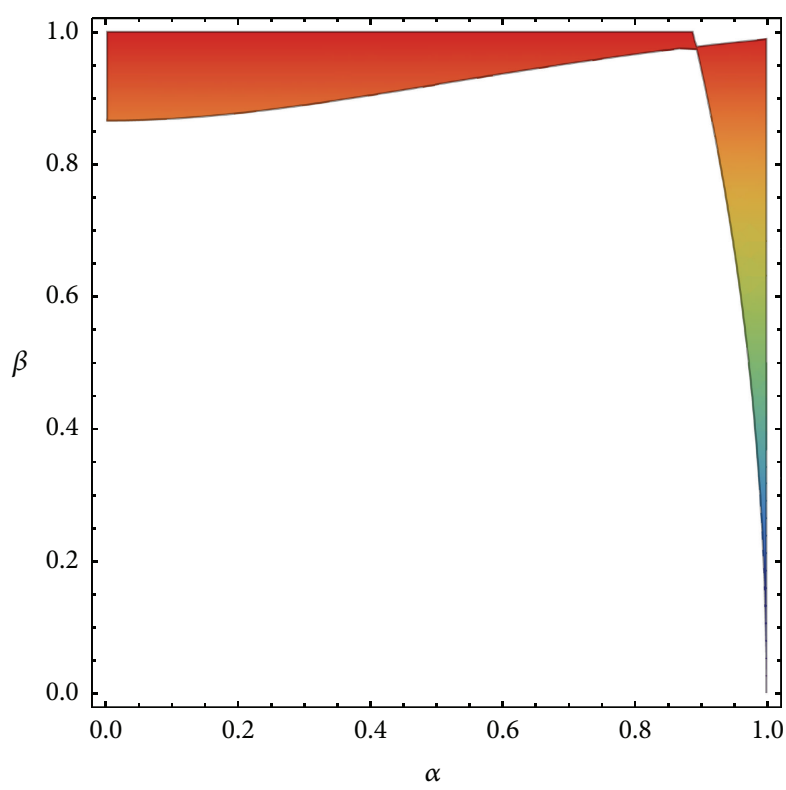

(c)

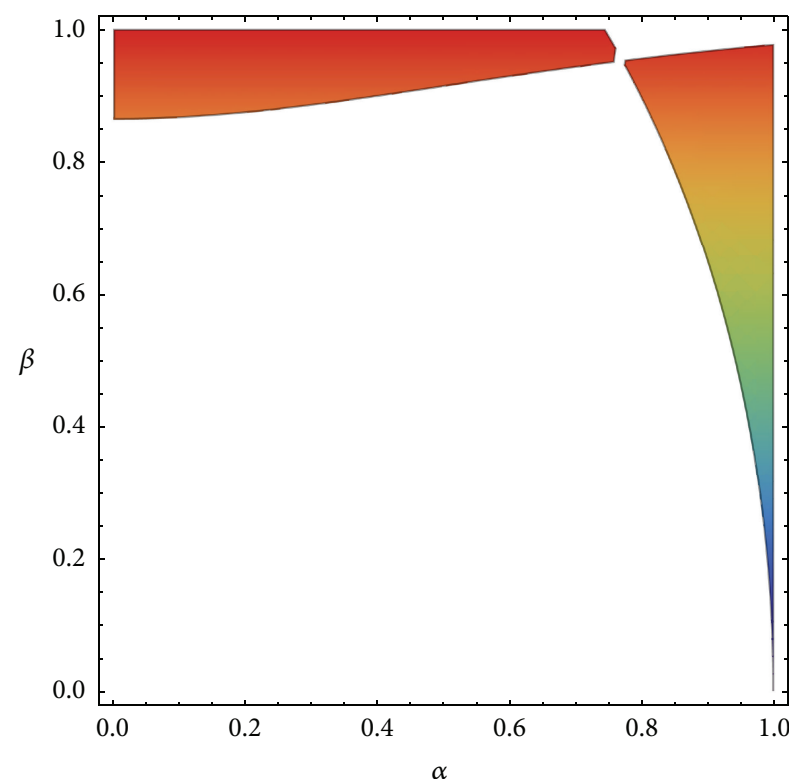

(b)

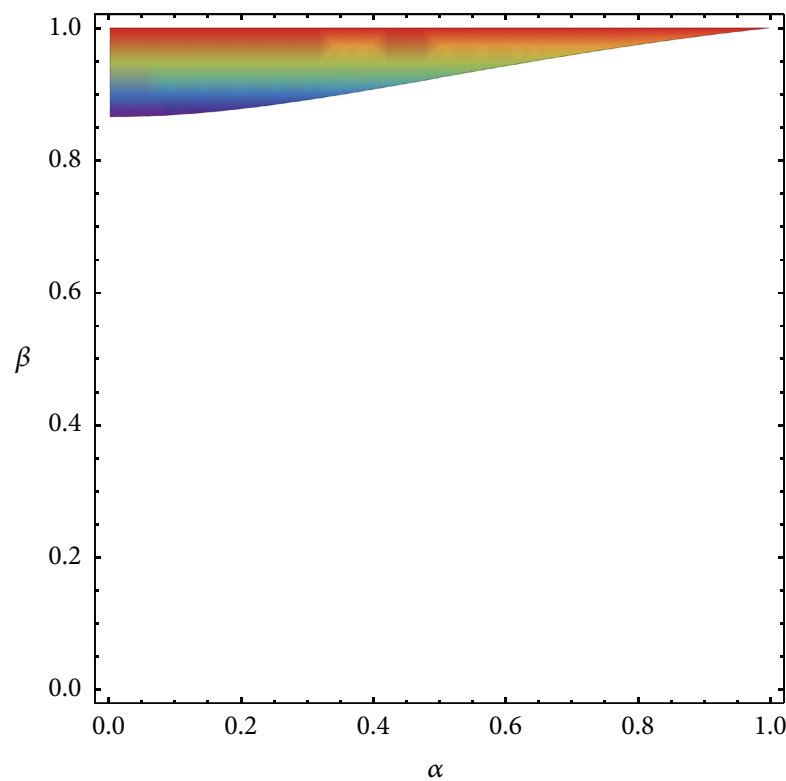

(d)

Figure 7: Cross sections of the central configurations region in the general case when $M, m_{0}>0$ and (a) $C=0.7$, (b) $C=0.8$, (c) $C=0.9$, and (d) $C=1$ give the case with four equal masses and a varying central mass.

configurations are possible for $\beta<0.42$. For $\beta>0.42$ there exists at least one $C$ such that both $m_{0}$ and $M$ are positive and form a $4+1$-body trapezoidal central configuration.

\section{Conclusions}

In this paper, we model isosceles trapezoidal four- and fivebody problems where four of the masses are placed at the vertices of an isosceles trapezoid and the fifth mass is placed at the center of mass of the system. To make use of the symmetries of the problem chosen we rewrite the position vectors of all the point masses as a linear combination of two vectors. This helped us halve the dimensions of the problem to a manageable level. Initially, we study a special case $\left(m_{i}=\right.$ $M, i=1, \ldots, 4)$ and form expressions for $m_{0}$ and $M$ as functions of $\alpha$ and $\beta$ which gives central configurations in the trapezoidal 5-body problems. CC regions are identified in the $\alpha \beta$-plane where both $m_{0}$ and $M$ are positive. In the same way we form expressions for $m_{0}$ and $M$ as functions of $\alpha, \beta$, and $C$ in the $4+1$-body cases which give central configuration regions in the trapezoidal $4+1$-body problems. In this case 


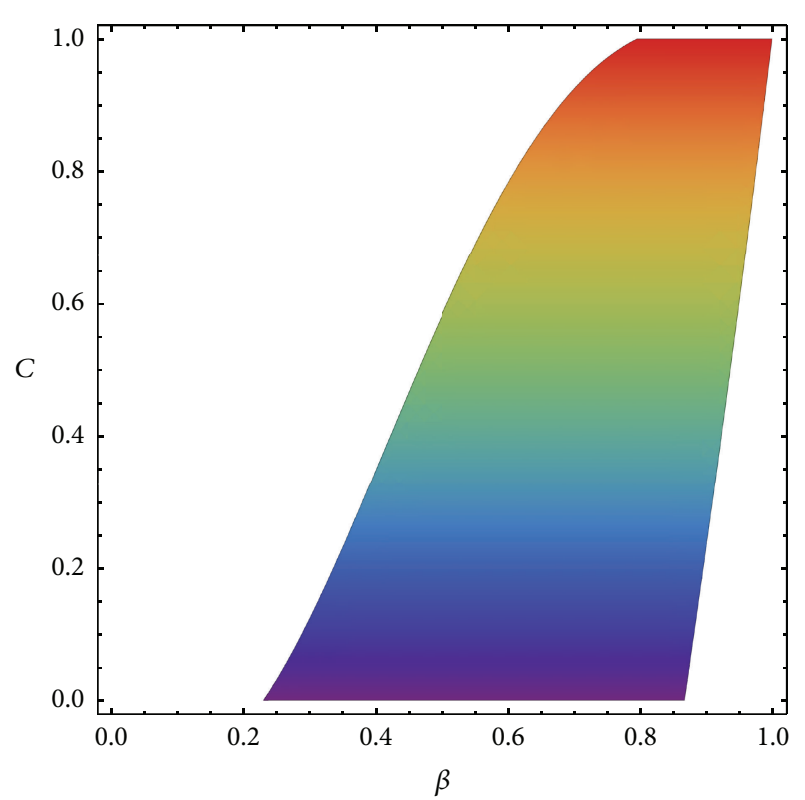

(a)

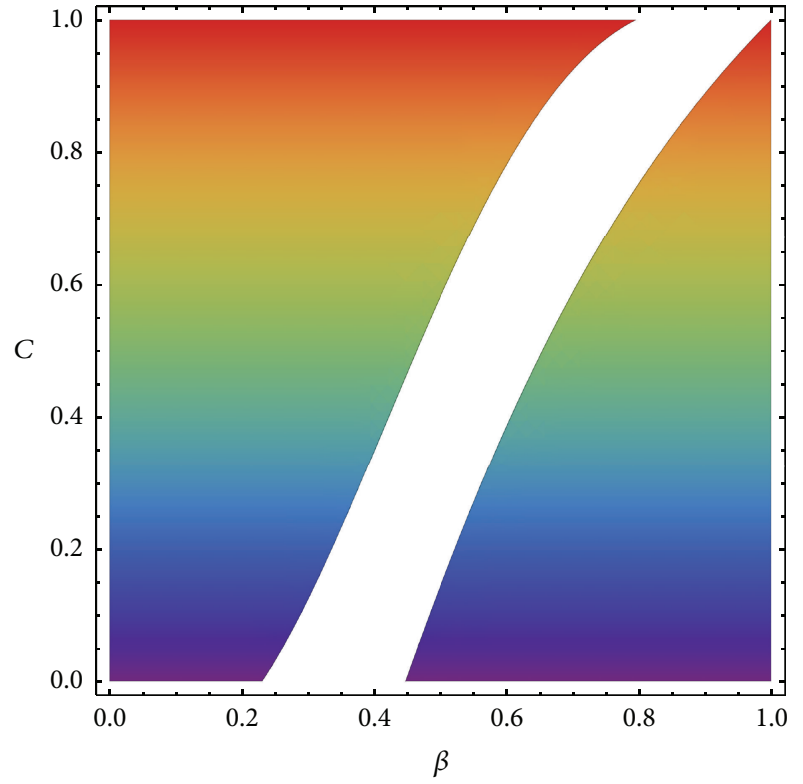

(b)

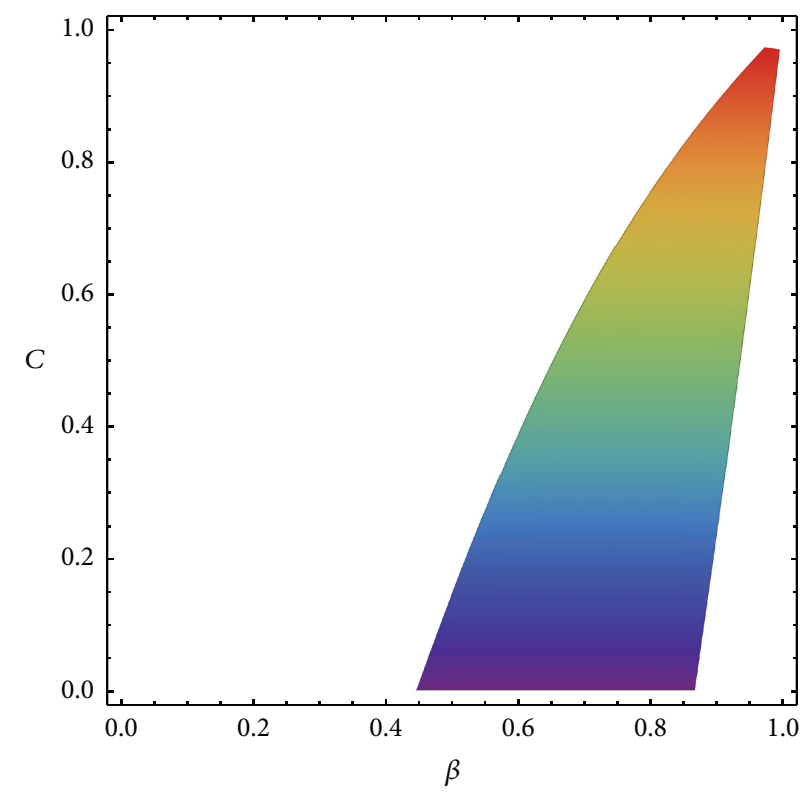

(c)

Figure 8: Central configurations region in the trapezoidal 5-BP when $\alpha=\beta$ (a) $m_{0}>0$, (b) $M>0$, and (c) $m_{0}>0$ and $M>0$.

there are two pairs of masses and a varying central mass. CC regions are identified both analytically and numerically. We also identify regions in the configuration space where no central configurations are possible for $m_{i}>0, i=0,1,2,3,4$, and $m_{0}>0$.

\section{Conflict of Interests}

The author declares that there is no conflict of interests regarding the publication of this paper.

\section{Acknowledgment}

The author thanks the Deanship of Research at the University of Hail, Saudi Arabia, for funding this work under Grant no. SM14014.

\section{References}

[1] A. Albouy and J. Llibre, "Celestial mechanics: dedicated to Donald Saari for his 60th birthday," Contemporary Mathematics, vol. 292, pp. 1-16, 2002. 
[2] M. Shoaib and I. Faye, "Collinear equilibrium solutions of fourbody problem," Journal of Astrophysics and Astronomy, vol. 32, no. 3, pp. 411-423, 2011.

[3] F. R. Moulton, "The straight line solutions of the problem of $\mathrm{N}$ bodies," Annals of Mathematics, vol. 12, no. 1, pp. 1-17, 1910.

[4] J. Palmore, "Classifying relative equilibria. II," Bulletin of American Mathematical Society, vol. 81, pp. 489-591, 1975.

[5] J. I. Palmore, "Collinear relative equilibria of the planar $n$-body problem," Celestial Mechanics, vol. 28, no. 1-2, pp. 17-24, 1982.

[6] K. E. Papadakis and S. S. Kanavos, "Numerical exploration of the photogravitational restricted five-body problem," Astrophysics and Space Science, vol. 310, no. 1-2, pp. 119-130, 2007.

[7] M. Kulesza, M. Marchesin, and C. Vidal, "Restricted rhomboidal five-body problem," Journal of Physics A: Mathematical and Theoretical, vol. 44, no. 48, Article ID 485204, 2011.

[8] A. Ollongren, "On a particular restricted five-body problem an analysis with computer algebra," Journal of Symbolic Computation, vol. 6, no. 1, pp. 117-126, 1988.

[9] T. J. Kalvouridis, "A planar case of the $\mathrm{n}+1$ body problem: the 'ring' problem," Astrophysics and Space Science, vol. 260, no. 3, pp. 309-325, 1998.

[10] V. V. Markellos, K. E. Papadakis, and E. A. Perdios, "The plane restricted five-body problem," in Proceedings of the 6th Joint European and National Astronomical Meeting: New Trends in Astronomy and Astrophysics (JENAM '97), pp. 1-15, Thessaloniki, Greece, July 1997.

[11] G. E. Roberts, "A continuum of relative equilibria in the fivebody problem," Physica D: Nonlinear Phenomena, vol. 127, no. 3-4, pp. 141-145, 1999.

[12] V. Mioc and C. Blaga, "A class of relative equilibria in the manev five-body problem," Hvar Observatory Bulletin, vol. 23, pp. 4148, 1999.

[13] M. Shoaib, B. A. Steves, and A. Széll, "Stability analysis of quintuple stellar and planetary systems using a symmetric fivebody model," New Astronomy, vol. 13, no. 8, pp. 639-645, 2008.

[14] M. Shoaib, A. Sivasankaran, and A. Kashif, "Central configurations in the collinear 5-body problem," Turkish Journal of Mathematics, vol. 38, no. 3, pp. 576-585, 2014.

[15] T. L. Lee and M. Santoprete, "Central configurations of the five-body problem with equal masses," Celestial Mechanics and Dynamical Astronomy, vol. 104, no. 4, pp. 369-381, 2009.

[16] M. Gidea and J. Llibre, "Symmetric planar central configurations of five bodies: Euler plus two," Celestial Mechanics and Dynamical Astronomy, vol. 106, no. 1, pp. 89-107, 2010.

[17] M. Marchesin and C. Vidal, "Spatial restricted rhomboidal fivebody problem and horizontal stability of its periodic solutions," Celestial Mechanics and Dynamical Astronomy, vol. 115, no. 3, pp. 261-279, 2013.

[18] T. Ouyang and Z. Xie, "Collinear central configuration in fourbody problem," Celestial Mechanics and Dynamical Astronomy, vol. 93, no. 1-4, pp. 147-166, 2005.

[19] L. F. Mello and A. C. Fernandes, "Co-circular and co-spherical kite central configurations," Qualitative Theory of Dynamical Systems, vol. 10, no. 1, pp. 29-41, 2011. 

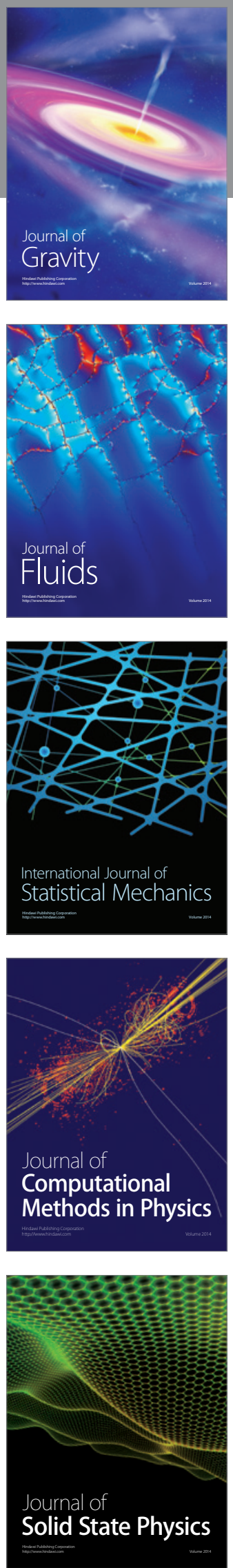

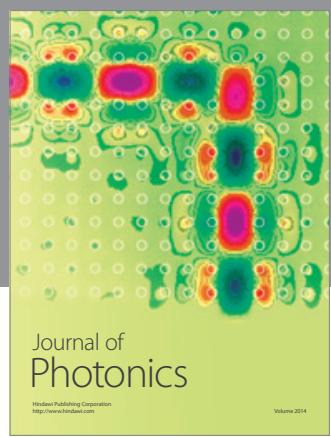

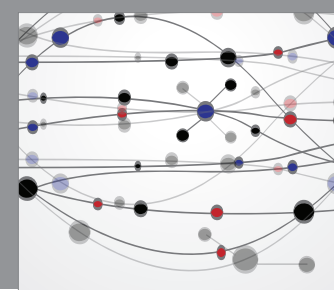

The Scientific World Journal

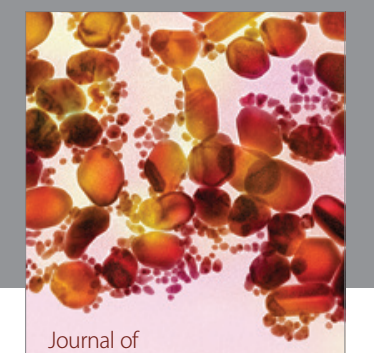

Soft Matter
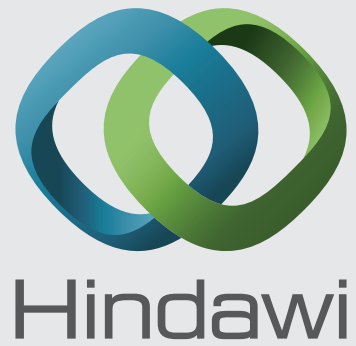

Submit your manuscripts at

http://www.hindawi.com
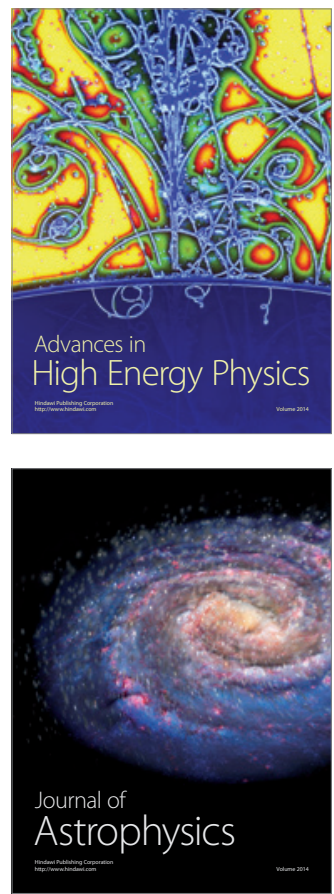
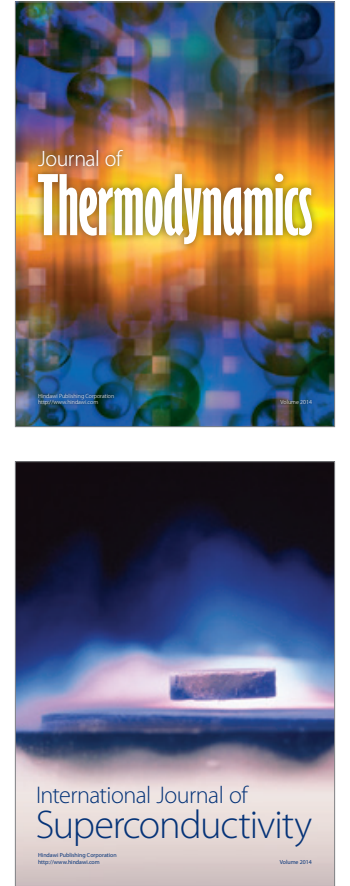
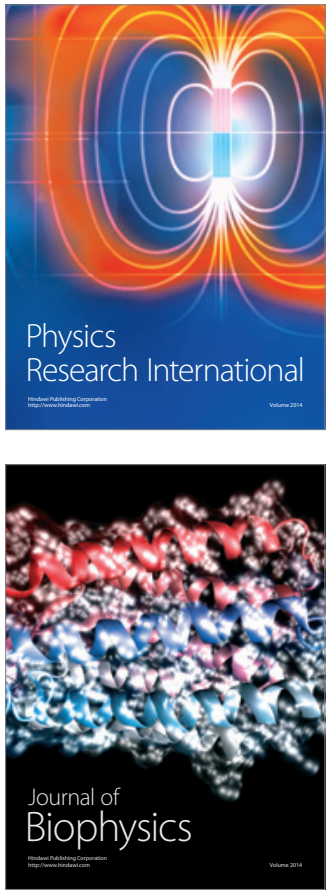
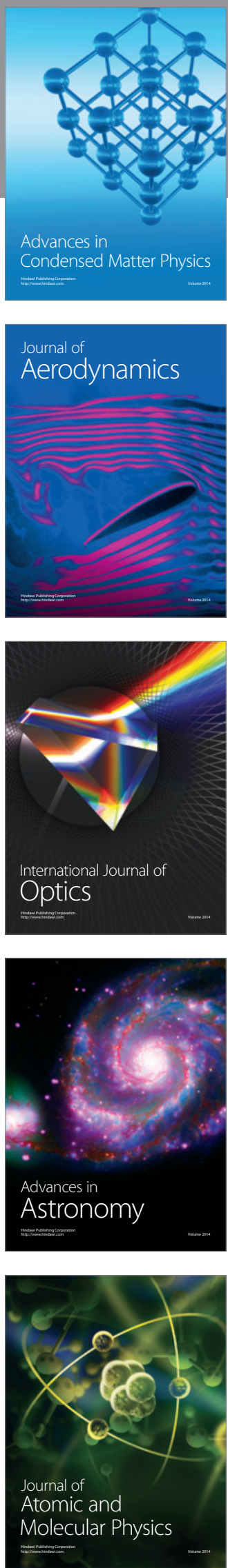